\title{
O professsor de educação física no Sistema Unico de Saúde: sua prática e resultados - estudo de revisão
}

\author{
Teacher of physical education System Unified Health: \\ your practice and results - review of study
}

Resumo

O estudo teve como objetivo realizar revisão sistemática da literatura referente ao trabalho do professor de Educação Física no Sistema Único de Saúde (SUS), com ênfase na sua prática e nos resultados que dela decorrem. As buscas foram realizadas nas bases de dados Google Acadêmico, Scielo, Pubmed e Biblioteca Virtual em Saúde. O processo de seleção resultou em 12 publicações para o estudo de revisão.As experiências indicam resultados positivos para a promoção da saúde e a qualidade de vida das pessoas, inclusive com redução dos gastos do setor saúde. A formação em Educação Física precisa se adequar para a realidade prática de trabalho do professor de Educação Física no SUS ampliando os espaços formadores das disciplinas e dos estágios.

Palavras-chave: Educação.EducaÇão Física. SaúdeColetiva.

\section{Abstract}

The study aimed to perform a systematic review of the literature on the work of physical education teacher in the Unified Health System (SUS), with emphasis on its practice and the results that flow from it. The searches were conducted on Google Scholar databases, Scielo,Pubmed and Virtual Health Library. The selection process resulted in 12 publications for the study review. The experiments indicate positive results for the promotion of health and quality of life, including lower spending in the health sector. Training in Physical Education needs to adapt to the practical reality of work of the teacher of physical education in the SUS expanding trainers spaces of disciplines and stages.

Keywords: Education. Physical Education. Public Health.

\section{INTRODUÇão}

Bruno de Souza Vespasiano

JoÃo Rogério de OLIVEIRA

Regina Zanella Penteado ${ }^{1}$

Marcelo de Castro Cesar ${ }^{\mathrm{I}}$ IUniversidade Metodista de

Piracicaba (UNIMEP), Piracicaba/

SP - Brasil
As doenças crônicas não transmissíveis (DCNT) são as principais causas de morte no mundo (acidente vascular cerebral, infarto, hipertensão arterial, câncer, diabetes e doenças respiratórias crônicas), tendo como principais fatores de risco: sedentarismo, alimentação não saudável, obesidade, tabagismo e álcool ${ }^{1}$. 
Destaca-se que a inatividade física e o aumento do sedentarismo, tanto em países desenvolvidos como em países em desenvolvimento, implica em riscos e impactos negativos na saúde pública ${ }^{1,2}$. $\mathrm{O}$ sedentarismo da população é considerado, portanto, um importante problema de saúde pública que mobiliza reflexões e esforços, nos meios acadêmico e profissional, na busca de alternativas e soluções ${ }^{3}$.

Neste cenário, a inserção do professorde Educação Física nas políticas e ações de promoção de saúde é merecedora de atenção, especialmente quando se considera que ela deve ser pautada por uma concepção ampla, abrangente, dinâmica e processual de saú$\mathrm{de}^{1,3}$. As propostas, os projetos e/ou programas voltados para o incentivo e a realização de práticas corporais e de atividade física ganham, assim, notoriedade ${ }^{4}$.

O Sistema Único de Saúde (SUS) configura a formulação política e organizacional dos serviços e ações de saúde pública/coletiva, oficializada na Constituição da República Federativa do Brasil de 1998 tendo sido implantado a partir da lei 8080 de $1990^{4}$.O SUS envolve um conceito amplo e abrangente de saúde, o que implica em investimentos na construção de políticas públicas saudáveis, na criação de ambientes favoráveis à promoção de saúde e na necessidade de reorientação da rede de assistências dos serviços de saúde e modelos de atenção, nas perspectivas da proteção e da promoção da saúde $^{4,5,6,7,8,9}$. O acesso à rede deve se dar a partir dos serviços de Atenção Primária à Saúde (APS), representados pelos atendimentos realizados por profissionais da saúde com formação generalista, nas Unidades Básicas de Saúde (UBS), com apoio de equipes interdisciplinares da Estratégia de Saúde da Família (ESF). As ações, desenvolvidas de forma integralizada em serviços com níveis de complexidade tecnológica crescente, devem ser orientadas para a promoção, a proteção e a recuperação da saúde das pessoas e contribuir para a melhoria das condiçõos e qualidade de vida, com aproximação dos serviços às necessidades de saúde e características de cada comunidade ${ }^{1,4-8,10}$.

Os Núcleos de Apoio à Saúde da Família (NASF), constituídos por equipes interdisciplinares que incluem o professor de educação física, foram criados a partir de 2008 com a perspectiva do cuidado compartilhado e da difusão de saberes, experiências e práticas, tendo como metas a melhor planejamento, organização e direcionamento do serviço realizado, a integralização do sistema, a ampliação do acesso ao atendimento e a melhoria dos resultados a ele relaciona$\operatorname{dos}^{5,8,9,11-15}$.

Cabe observar que uma das estratégias de atendimento preconizadas pelo NASF envolve a realização de práticas corporais e de atividade física para promover a saúde e melhorar a qualidade de vida da população - o que afirma a importância do professor de educação física integrar as equipes do NASF. ${ }^{5,11}$.

Uma revisão sobre a inserção e atuação do professor de Educação Físicana atenção básica à saúdeevidenciou contratos temporários que não viabilizam um trabalho de continuidade. Mostrou que a atuação do profissional de Educação Física na atenção básica está predominantemente centrado em pessoas idosas e/ou portadoras de Doenças Crônicas Não Transmissíveis (DCNT) e no desenvolvimento de caminhadas e atividades lúdicas e de fortalecimento muscular. O estudo conclui que a fragilidade da formação em Educação Física, pouco direcionadapara a interdisciplinaridade e para 
atuação profissional junto a grandes grupos populacionais, não contempla as demandas da atenção básica ${ }^{12}$.

O estudo teve como objetivo realizar revisão sistemática da literatura referente ao trabalho do professor de Educação Física no Sistema Único de Saúde (SUS), com ênfase na sua prática e nos resultados que dela decorrem. Leva-se em conta o lócus de inserção e as unidades e equipes às quais o profissional se vincula, com ênfase no recorte das práticas realizadas e dos resultados que delas decorrem.

\section{MATERIAL E MÉTODOS}

O estudo se refere a uma revisão sistemática, em que a literatura referente a um determinado tema ou questão que passa a compor a revisão e a análise não é escolhida de forma aleatória, mas sim a partir de um processo de seleção que se dá a partir de critérios definidos e pré-estabelecidos, bem como processos de inclusões e de exclusões ${ }^{15,16}$.

O processo envolve as etapas: 1) Definição da pergunta ou questão/problema; 2) Definição de estratégias de busca e seleção (critérios de inclusão/exclusão); 3) Busca; 4) Seleção das publicações mediante aplicação dos critérios; e 5) Análise das publicações selecionadas para revisão ${ }^{16}$.

Algumas questões/problemas orientam a presente pesquisa: Como vem se dando a inserção e a atuação do professor de Educação Física no Sistema Único de Saúde (SUS)? O professor de Educação Física tem, efetivamente, integrado as equipes de trabalho das UBS, das ESF e/ou dos NASFs? Quais as contribuições do professor de Educação Física junto ao SUS? Quais as práticas realizadas por este profissional no âmbito do SUS?
As buscas foram realizadas no mês de novembro de 2015, nas seguintes bases de dados: Google acadêmico, Scielo, Pubmed e Biblioteca Virtual em Saúde (BVS).

Os termos empregados nas buscas foram: Educação Física (e) Sistema Único de Saúde / SUS; Educação Física (e) Saúde da Família; Educação Física (e) Núcleo de Atenção à Saúde da Família / NASF. Tambémforamempregadosostermosem ingles: Physical Education (and) Unified Health System; Physical Education (and) Family Health; Physical Education (and) Family Health Support Nuclei.

Foram incluídas aceitas publicações em inglês e português, sem restrição ou delimitação quanto ao período de tempo.

Feita a busca inicial, uma seleção preliminar envolveu a identificação das publicações duplicadas, com exclusões das repetidas, mantendo-se apenas um elemento.

Após esta etapa deu-se início o processo de aplicação dos critérios de seleção, o que implica em inclusões e exclusões. Para a seleção dos estudos, foram utilizados os parâmetros propostos pelo Checklist Prisma ${ }^{13}$.

A partir dos títulos e/ou resumos, buscou-se identificar as publicações que se aproximavam e as que se distanciavam do escopo do estudo - sendo que aquelas que se distanciaram foram excluídas.

Como principal critério de inclusão foram consideradas as publicações que apontavam a inserção do professor de Educação Física no SUS; sendo priorizadas as publicações com texto completo, que configuram artigos em periódicos científicos (artigos originais, relatos de experiência, estudos teóricos e/ou de revisão).

Foram excluídas todas as publicações que escapassem do escopo do estudo e também aquelas que se tratavam dos documen- 
tos oficiais e/ou políticas do Ministério da Saúde, editoriais, cartas, resenhas, resumos em anais de evento, monografias, dissertações, teses e/ou matérias de jornais.

Uma vez selecionadas as publicações para a revisão, estas foram lidas integralmente e analisadas, tendo em vista identificar, em cada uma delas, os conteúdos que possibilitam caracterizar a inserção do professor de Educação Física no Sistema Único de Saúde.

Os dados e conteúdos das publicações referentes à inserção do professor de Educação Física no SUS foram classificados em categorias e apresentados na tabela 1, referentes a: autores, ano da publicação, unidade/ serviço de inserção, práticas realizadas pelos professores de Educação Física e resultados e/ou contribuições da inserção do profissional no SUS.

\section{Resultados}

As buscas iniciais resultaram em 1470 publicações. Feita a exclusão dos repetidos $(n=523)$ resultaram 947 publicações. Sobre elas foram aplicados os critérios de seleção (inclusões e exclusões), o que resultou na exclusão de 933 publicações e na seleção final de 13 publicaçõespara compor o estudo de revisão sistemática.

O fluxograma a seguir sintetiza este processo (Figura 1).

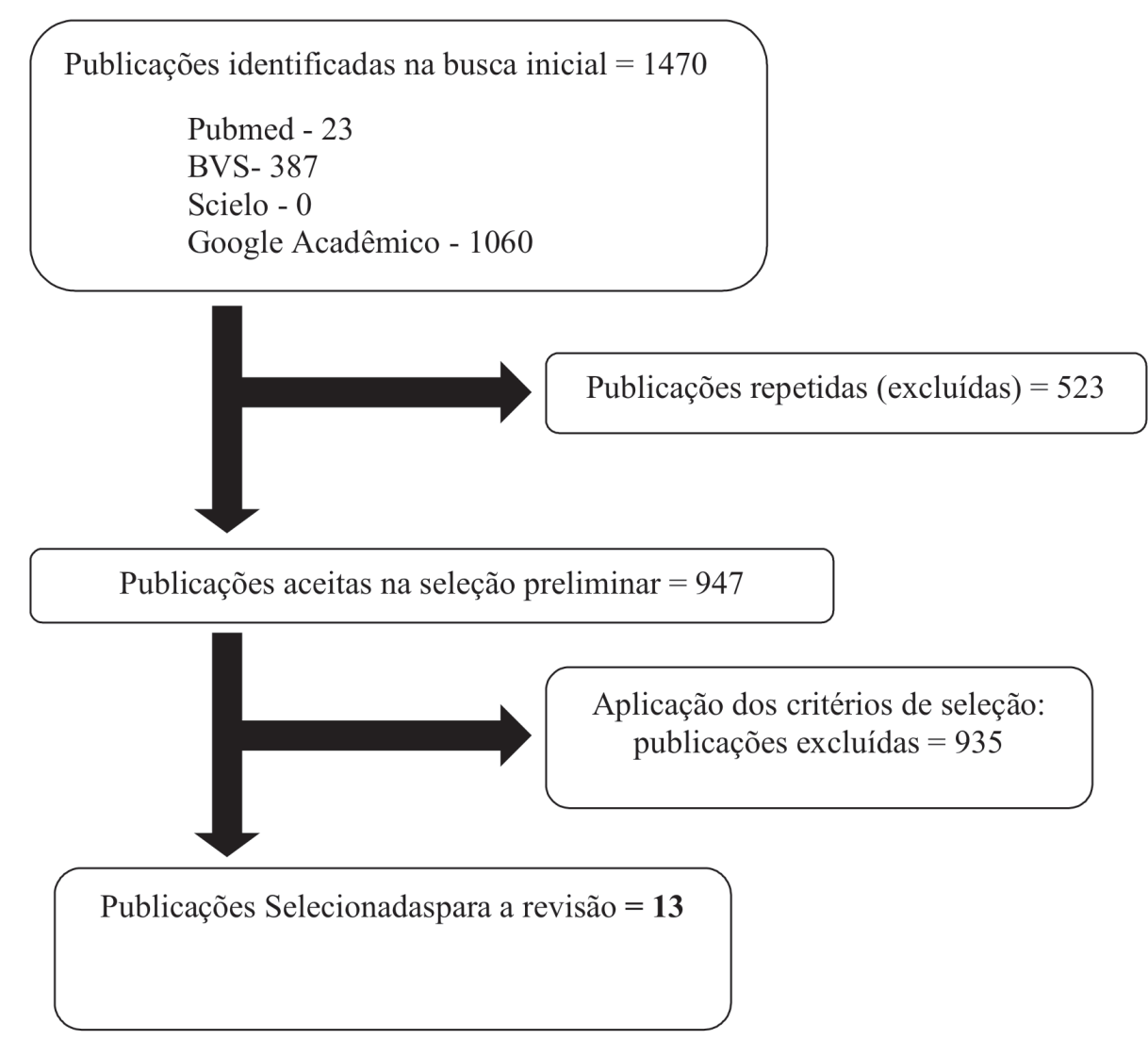




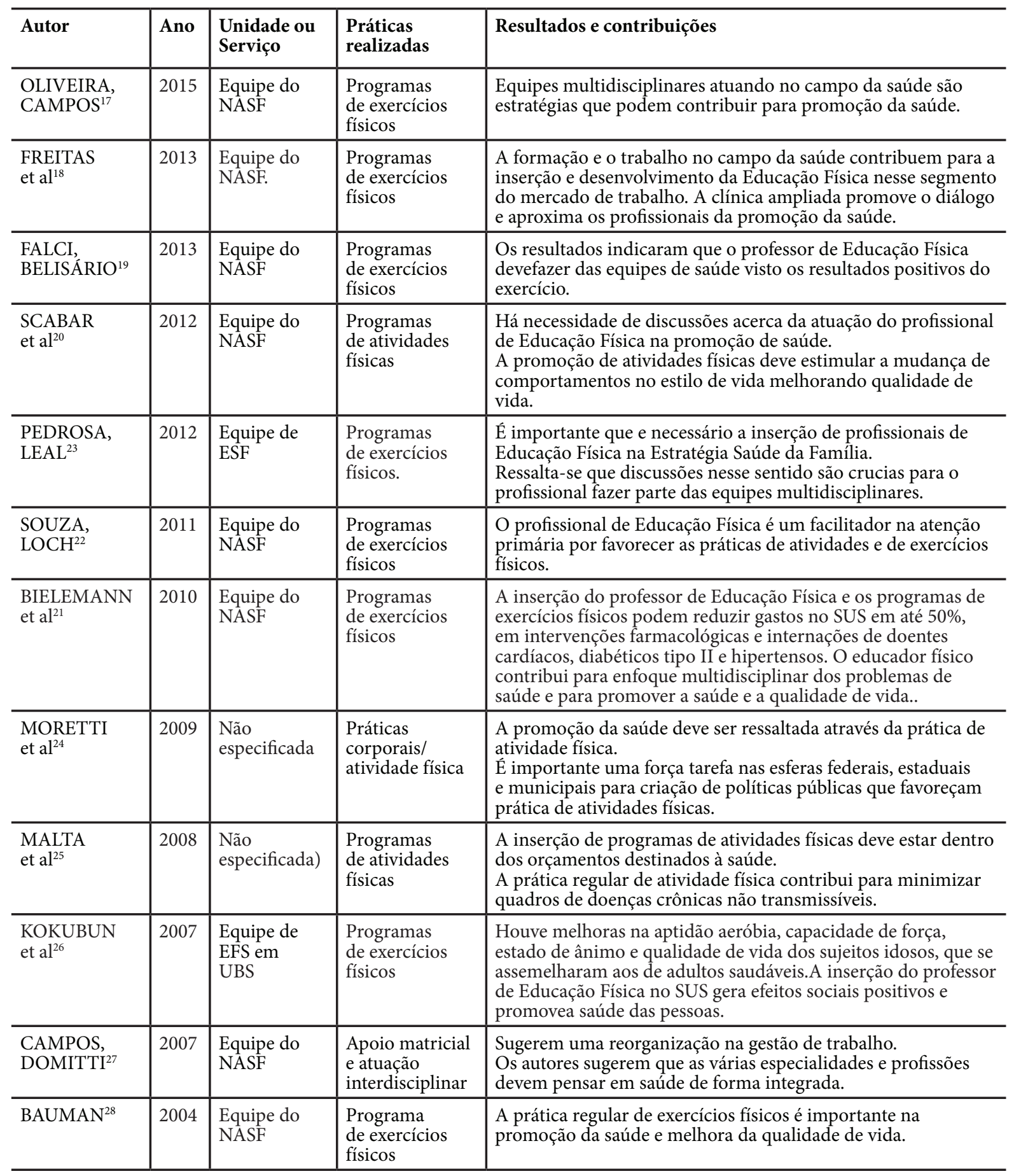

TABELA 1.Caracterização e distribuição das publicações que tratam da inserção do professor de Educação Física no SUS segundo: autores/ano, unidade/serviço, práticas realizadas e os resultados e contribuições. 


\section{Discussão}

A tabela 1 mostra que há poucas publicações voltadas para a inserção do professor de Educação Física no Sistema Único de Saúde e demonstram a necessidade de ampliação das investigações e dos relatos de experiências concernentes a esta questão.

Este estudo de revisão mostra que a inserção do professor de Educação Física no SUS é ainda incipiente e que as práticas desse profissional vêm se dando junto às equipes de Estratégia de Saúde da Família e Núcleos de Atenção à Saúde da Família, com atuações que focalizam prioritariamente os programas de exercícios físicos e de atividades físicas.

Os efeitos e resultados da inserção do profissional de Educação Física no SUS foram, sempre, avaliados como positivos para os segmentos das comunidades e populações envolvidas e geradores de benefícios à promoção da saúde geral e da qualidade de vida e, inclusive, de economias e de redução de gastos do sistema ao sistema ${ }^{26-28}$.

O período das publicações remonta há pouco mais de uma década, a partir do ano de 2004. Nota-se que algumas publicações ocorreram antes mesmo da criação do NASF, o que determinou a inserção do educador físico no âmbito do SUS ${ }^{26-28}$. Entretanto é possível perceber que, entre os anos de 2004 e 2015, praticamente não houve mudanças no tipo de prática realizada.

As práticas do professor de Educação Física no SUS são configuradas, basicamente, pela orientação de programas de atividades físicas/corporais e exercícios físicos. Um único estudo ${ }^{27}$ destaca a prática de apoio matricial e atuação interdisciplinar, entretanto sem maiores detalhamentos.
Os exercícios físicos são formas sistematizadas de programas e devem ser desenvolvidas por profissionais de Educação Física no âmbito da saúde ${ }^{23}$. A possibilidade da implantação de Programas de Exercícios Físicos contribui para a promoção da saúde e a melhora da qualidade de vida das comunidades envolvidas, com diminuição do consumo de fármacos e economia e redução dos investimentos e gastos públicos em saúde, em conformidade com o que preconizam os documentos e as políticas de saúde ${ }^{22,27}$.

As experiências de inserção do professor de Educação Física no SUS se apresentam, portanto, em conformidade com a literatura, que considera que a prática regular de exercícios físicos é importante para a melhora da aptidão física, para a realização das atividades de vida diária com maior efetividade e qualidade de vida, além de prevenir doenças como obesidade, hipertensão arterial, diabetes entre outras, promovendo a saúde ${ }^{22-27}$.

Além disto, as ações que envolvem a ampliação e efetivação da atividade física para a promoção da saúde e qualidade de vida precisam ser incentivadas por meio de implantação de políticas públicas municipais e estaduais nas quais o professor de Educação Física deve estar envolvido ${ }^{14,21,23,24}$.

Neste sentido, o presente estudo de revisão mostra que a inserção do professor de Educação Física corresponde, exatamente, às expectativas primárias de atuação para este profissional. Os resultados são condizentes a outros estudos de revisão, que identificaram como principais práticas a condução de exercícios físicos e de ginásticas e caminhadas ${ }^{29}$

Não foram observadas práticas inovadoras. As abordagens se repetem, assim como as conclusões dos estudos realizados. 
Os cursos de Licenciatura e de Bacharelado em Educação Física devem se ater às possibilidades de um mercado promissor, de atuação dos educadores físicos, representado pelo SUS, para melhor orientar suas práticas educacionais e formativas ${ }^{24}$.

Entretanto uma melhor orientação da formação do professor de Educação Física para atuar em Saúde Coletiva requer um estreitamento do diálogo entre os cursos/as universidades e os serviços e a realização de pesquisas que contribuam para gerar subsídios para a discussão das especificidades e das possibilidades de ampliação da atuação profissional neste campo, levando em conta as necessidades e as demandas populacionais e comunitárias e as vias de diálogo e de articulação interdisciplinar.

Novos estudos devem ser realizados, a fim de possibilitar melhor caracterizar as condições de inserção do professor de Educação Física no SUS e, assim, melhor subsidiar as discussões orientadas para uma formação de qualidade dos professores e profissionais de Educação Física no que se refere ao trabalho no SUS ${ }^{22}$.

O presente estudo de revisão evidencia, portanto, que as publicações que versam sobre as experiências de inserção do profissional/professor de Educação Física no SUS não vêm acrescentando elementos significativos para a potencialização da caracterização da atuação deste profissional no âmbito do SUS, nem mesmo para uma reconfiguração das suas práticas e/ou uma consolidação de uma atuação interdisciplinar deste profissional em Saúde Coletiva.

A literatura voltada para a atuação do professor de Educação Física no SUS está concentrada em registrar a inserção do pro- fissional e o resultado positivo junto à saúde dos usuários. A atenção não se encontra voltada para o estudo das práticas profissionais da Educação Física no contexto da Saúde Coletiva. Neste sentido, algumas questões podem indicar possibilidades para investigações futuras e discussão de aspectos ainda pouco explorados: Como podem ser caracterizadas as práticas do professor de Educação Física no âmbito do SUS? Qual tem sido o papel desempenhado pelo profissional de Educação Física nas equipes das UBS, ESF e NASF? Quais são os segmentos da população/comunidade e os ciclos de vida que correspondem aos sujeitos envolvidos na maioria das práticas da Educação Física do SUS? Quais segmentos ainda estariam fora do foco de atenção?

As publicações selecionadas não permitiram evidenciar a ocorrência de avanços, de ampliações ou de re-configurações das práticas do professor de Educação Física em função da inserção deste profissional no âmbito do SUS.

Nota-se, assim, que os efeitos da inserção do profissional de Educação Física no SUS (ainda que com resultados positivos e favoráveis para a promoção da saúde da população e para o próprio sistema de saúde) são ainda tímidos e fragilizados.

As experiências do profissional de Educação Física nos serviços e equipes interdisciplinares das UBS, ESF e NASF não têm sido potentes em desenvolver novas práticas. Esta reflexão deve ser levada aos espaços sociais de formação.

Certamente o profissional de Educação Física poderia contribuir com várias outras ações e formas de atenção aos usuários do SUS, que não somente aquelas restritas às 
atividades físicas e exercícios físicos - ainda que sejam elas imprescindíveis.

Estudos atuais mostram que a formação em Educação Física para a atuação no SUS sofre um distanciamento em relação aos conteúdos da saúde pública e saúde coletiva ${ }^{29}$

A formação teórico/prática em Educação Física deveria contemplar experiências interdisciplinares junto a outros cursos (Fisioterapia, Nutrição, Fonoaudiologia, Farmácia, Enfermagem, Pedagogia e outros) e estágios no SUS que propiciassem o desenvolvimento e a consolidação de ações coletivas e práticas consonantes com uma perspectiva ampla, abrangente e dinâmica de saúde e com a promoção da saúde pautada pela integralidade e interdisciplinaridade.

Algumas experiências pontuais avançam neste sentido, em disciplinas de Saúde Coletiva ${ }^{30}$ oferecidas em tronco comum a alunos de diversos cursos da área de saúde de determinadas Faculdades/Universidades. Nestas experiências, os alunos de Educação Física são levados a uma análise ampla dos problemas de saúde, bem como ao planejamento conjunto, elaboração e desenvolvimento de ações interdisciplinares em saúde coletiva que envolvem temáticas variadas e abrangentes para além das preocupações específicas com a atividade física e os exercícios físicos.

Faltam, contudo, relatos mais apurados destas experiências formativas, bem como estudos aprofundados de acompanhamento dos efeitos formativos que este tipo de experiência educacional propicia aos seus alunos e, também, carecem investigações das repercussões e das implicações de disciplinas como estas, na expansão das práticas profissionais desenvolvidas no SUS pelos egressos dos cursos de Educação Física e nas maneiras de engajamento interdisciplinar.

A formação em Educação Física precisa se adequar às possibilidades do trabalho interdisciplinar em Saúde Coletiva, com expansão e reconfiguração das práticas profissionais a partir da criação de espaços de reflexão e discussão em disciplinas teóricas, teórico-práticas e estágios, seja na formação inicial ou continuada.

\section{Conclusões}

O Sistema Único de Saúde se apresenta como um campo promissor para a inserção e atuação profissional do professor de Educação Física; no entanto esta revisão mostrou que há poucas publicações que versam sobre a temática. Além disto, as publicações existentes não se mostram atentas à discussão das práticas realizadas.

A atuação do profissional de Educação Física no âmbito do SUS, restrita às práticas de incentivo à realização de atividades físicas e exercícios físicos, sugere um sub-aproveitamento das possibilidades de inserção e engajamento nas questões de Saúde Coletiva. As experiências do profissional de Educação Física nos serviços e equipes interdisciplinares das UBS, ESF e NASF não têm sido, ainda, potentes em inovar as práticas de modo a consolidar formas de contribuição mais amplas da Educação Física para a Saúde Coletiva, que se apresentem mais afinadas com das diretrizes do SUS, como uma concepção ampla, abrangente e dinâmica de saúde e a perspectiva de promoção da saúde pautada pela integralidade e pela interdisciplinaridade.

Há muito a avançar, na formação do professor de Educação Física, quando se trata da atuação em Saúde Coletiva 


\section{Colaboração dos autores}

Todos os autores participaram do planejamento e desenvolvimento do estudo, o que envolve coleta, tratamento e análise de dados, a elaboração, a redação e revisão.

\section{Conflito de interesses}

Não há conflitos de interesse.

\section{REFERENCIAS}

1. Nahas MV. Atividade Física, saúde e qualidade de vida:conceitos e sugestões para um estilo de vida mais ativo. 5 ed. Londrina: Miograf; 2010.

2. Vespasiano BS, Mella, A, Montebelo MIL, Cesar MC. Composição Corporal e do Nível de Atividade Física de Alunos do Ensino Médio em uma Escola pública e uma Particular de Itapeva/SP. RevistaConexões, (11):31-59.2013;

3. Matsudo SM, Matsudo VR, Andrade DR. Physical activity promotion: experience and evaluation of agita São Paulo program using the ecological mobile model. Physical Activity Health.2004; (1):81-97.

4. Brasil MS. Ministério Da Saúde. ABC do SUS: Doutrina e Princípios. Brasília, 1990.

5. Brasil MS. Secretaria de Políticas de Saúde. Programa Nacional de Promoção da Atividade Física "Agita Brasil": Atividade física e sua contribuição para a qualidade de vida. Cadernos de Saúde Pública.2002; (36):254-256.

6. Brasil MS. Ministério da Saúde. Portaria no 648 de 28 de março de 2006. Aprova a Política Nacional de Atenção Básica, 2006.

7. Brasil MS. Portaria no 154 de 24 de janeiro de 2008. Cria os núcleos de apoio à Saúde da FamíliaNASF. Orientações para implantação dos núcleos. Brasília: Ministério da Saúde, 2008.

8. Brasil MS. Portaria n.719, de 07 de abril de 2011. Institui o Programa Academia da Saúde no âmbito do Sistema Único de Saúde. Brasília: Ministério da Saúde, 2011.

9. Brasil MS. Portaria no 3.124 de 28 de dezembro de 2012. Redefine os parâmetros de vinculação dos Núcleos de Apoio à saúde da Família (NASF) Modalidades 1 e 2 às Equipes Saúde da Família e /ou Equipes de Atenção Básica para populações específicas, cria a Modalidade NASF 3, e da outras providências. Brasília: Ministério da Saúde, 2012.

10. Campos GWS, Gutiérrez AC, Guerreiro AVP, Cunha GT. Reflexões sobre a atenção básica e a estratégia de saúde da família. In: Campos GWS, Gutiérrez, AC, Guerreiro, AVP. (org.). Manual de práticas de atenção básica: saúde ampliada e compartilhada. São Paulo: Hucitec, 2008.

11. Brasil MS. Diretrizes do NASF- Núcleo de Apoio à Saúde da Família. Ministério da Saúde. Secretaria de Atenção à Saúde. Departamento de Atenção Básica. Brasília: Ministério da Saúde, 2009.

12. Rodrigues JD, Ferreira DKS, Silva PA, Caminha IO, Junior JCF. Inserção e atuação do profissional de educação física na atenção básica à saúde: revisão sistemática. Revista Brasileira de Atividade Física e Saúde.2013;(18):5-15

13. Freitas FF. A Educação Física no serviço público de saúde. São Paulo: Hucitec, 2007.

14. Pelicioni MCF, Pelicioni AF, Toledo RF. A educação e a comunicação para a Promoção da Saúde. In: Rocha AA, Cesar CLG. Saúde pública: bases conceituais. São Paulo: Atheneu, 2008.

15. Nahas MV, Garcia LMT. Um pouco de história, desenvolvimentos recentes e perspectivas para a pesquisa em Atividade Física e Saúde no Brasil. Revista Brasileira de Educação Física e Esporte.2010; (24):135-148.

16. Altman DG, Moher D, Liberati A, Tetzlaff J. Preferredreportingitems for systematicreviewsand meta-analyses: The PRISMA Statement. Journal of the America Physical Therapy Associaton.2009; (89):873-880. 
17. Oliveira MM, Campos GWS. Apoio matricial e institucional: analisando suas construções. Ciência e Saúde Coletiva.2015; (20):229-238.

18. Freitas FF, Carvalho YM, Mendes VM. Educação Física e Saúde: aproximações com a "Clínica Ampliada”. Revista Brasileira de Ciências do Esporte.2013; (35):639-656.

19. Falci DM, Belisário SA. The position of physical education professionals within primary healthcare and the challenges in their training.Interface.2013; (17):885-899

20. Scabar TG, Pelicioni AF, Pelicioni MCF. Atuação do Profissional de Educação Física no Sistema Único de Saúde: uma análise a partir da Política Nacional de Promoção da Saúde e das Diretrizes do Núcleo de Apoio à Saúde da Família- NASF. Journal of the Health Sciences Institute.2012; (30):411-418.

21. Bielemann RM, Knuth AG, Hallal PC. Atividade física e redução de custos por doenças crônicas ao Sistema Único de Saúde. Revista Brasileira de Atividade Física e Saúde.2010; (15):9-16.

22. Souza SC, Loch MR. Intervenção do profissional de educação física nos núcleos de apoio à saúde da família em municípios do Norte do Paraná. Revista Brasileira de Atividade Física e Saúde, 2011; (16):5-10.

23. Pedrosa OP, Leal AF. A inserção do profissional de Educação Física na estratégia de saúde da família em uma capital do norte do Brasil. Revista Movimento.2012; (18):235-253.

24. Moretti AC, Almeida V, Westphal MF, Bógus CM. Práticas Corporais/ Atividade Física e Políticas Públicas de Promoção de Saúde. Revista Saúde e Sociedade.2009; (18):346-354.

25. Malta DC, Castro AM, Cruz DKA, Gosh CS. A Promoção da Saúde e da Atividade Física no Sistema Único de Saúde. Revista Brasileira de Atividade Física e Saúde.2008; (13):24-27.

26. Kokubun E, et al. Programa de Atividade Física em Unidades Básica de Saúde: Relato de Experiência no Município de Rio Claro- SP. Revista Brasileira de Atividade Física e Saúde. 2007; (12):45-53.

27. Campos GWS, Domitti AC. Apoio matricial e equipe de referência: uma metodologia para gestão do trabalho interdisciplinar em saúde. Cadernos de Saúde Pública. 2007; (23):399-407.

28. Bauman, AF. Updating the evidence that physical is good for health: an epidemiological review 2000-2003. Journal of Science and Medicine in Sport.2004: (1): 6-19.

29. Mendonça, P.H.L. Educação física na atenção básica do SUS: uma revisão integrativa. Dissertação. Universidade Federal de Goiás, 2016. Disponível em: http://repositorio.bc.ufg.br/tede/handle/tede/6178 acesso em: 5/8/2017.

30. Oliveira C.M.D.; Penteado R.Z.; Garbelini D. Promoção da saúde do estudante universitário: interdisciplinaridade e integralidade no contexto das práticas educacionais da disciplina Saúde Coletiva. Anais, 14 Mostra Acadêmica da Unimep, Piracicaba. Disponível em: http://unimep.br/ anexo/adm/25102016150307.pdf Acesso em: 2/08/2017 - p.234

\section{DADOS DOS AUTORES}

\section{Bruno de Souza Vespasiano}

Doutor em Ciências do Movimento Humano pela Universidade Metodista de Piracicaba. Professor na Faculdade de Ciências Sociais e Agrárias de Itapeva. Itapeva/SP - Brasil. brunovespasiano@msn. com

\section{JoÃo Rogério de OliveIRA}

Mestre em Ciência do Movimento Humano pela Universidade Metodista de Piracicaba. Docente na União das Instituições Educacionais de São Paulo. Tietê/SP - Brasil. jrcoren@yahoo.com.br 


\section{Regina Zanella Penteado}

Doutora em Saúde Pública pela Universidade de São Paulo. Docente na Universidade Metodista de Piracicaba. Piracicaba/SP - Brasil. rzpenteado@unimep.br

\section{Marcelo de Castro Cesar}

Doutor em Ciências pela Universidade Federal de São Paulo. Docente na Universidade Metodista de Piracicaba.Piracicaba/SP - Brasil.maccesar@unimep.br

Submetido em: 28-06-2017

Aceito em: 7-8-2017 